\title{
AUTOMATED EXPLORATION OF DESIGN SOLUTION SPACE APPLYING THE GENERATIVE DESIGN APPROACH
}

\author{
Li, Haibing; Lachmayer, Roland \\ Leibniz Universität Hannover
}

\begin{abstract}
Design is a complex problem-solving activity that transforms design restrictions and requirements into a set of constraints and explores the feasible solutions to satisfy those constraints. However, design solutions generated by traditional modeling approaches are hardly to deal with such constraints, particularly for the exploration of the possible design solution space to enhance the quality of the design outputs and confront the evolving design requirements. In this regard, the Generative Design Approach (GDA) is considered as an efficient method to explore a large design solution space by transforming the design problem into a configuration problem. Fundamentally, GDA explores and stores all the necessary knowledge through a design skeleton and a set of design elements. Thus, design solution space is easily explored by configuring variable design elements via iterative design processes. Further, the output model is not only a design solution but also a design concept that designers could manipulate to explore unconsidered design configurations. Finally, a crank creation as a running example confirmed that GDA provides concrete aids to enhance the diversity of design solutions.
\end{abstract}

Keywords: Computer Aided Design (CAD), Product modelling / models, Design methods, Generative design approach, Design solution space exploration

Contact:

Li, Haibing

Leibniz Universität Hannover

Mechanical Engineering

Germany

li@ipeg.uni-hannover.de

Cite this article: Li, H., Lachmayer, R. (2019) 'Automated Exploration of Design Solution Space Applying the Generative Design Approach', in Proceedings of the 22nd International Conference on Engineering Design (ICED19), Delft, The Netherlands, 5-8 August 2019. DOI:10.1017/dsi.2019.114 


\section{INTRODUCTION}

Product development often involves a compound set of objectives and constraints such as abstract notions of function and aesthetic, performance, design requirements and costs. With these constraints, engineering design is characterized by a small range of possible solutions, which means that product development also implies a process of exploring design solutions (Alfaris, 2009). In this context, the design activity can be seen as an iterative process that evolves from abstract ideas to more complex and concrete solutions, attending to the constraints and embodying variable design requirements (Bodein $e t$ al., 2014). This process advances and backtracks as many times as necessary in order to achieve a good solution to the design problem. Thus, design tools or approaches have to embrace changes in order to truly support and accomplish such design tasks. Otherwise, it requires too much time and effort to modify models or even treat them as disposable when changes are needed (Gembarski et al., 2017).

Computer-aided design systems (CAD-systems) are an important enabler in facing such design challenges to support engineers in finding a solution faster, contributing to increasing their efficiency (Gembarski et al., 2015). Unfortunately, such CAD-systems are currently being used just as a more efficient method that allow designers to easily create and relate the parts of a 3D model that reflects various design requirements, other than an approach that searches for design solutions and helps the engineers at handling design changes, particularly for the exploration of different solutions to adapt the evolving requirements (Weber, 2011). That means that such systems have limitations in the ability to make modifications within an interactive environment, requiring a model to be recreated with different attributes and parameters if changing geometry configuration or topology are needed.

\subsection{Motivation}

Based on the aforementioned problems, several approaches have been developed in contemporary CADsystems, e.g., parametric design (Monedero, 2000), feature-based design (Shahin, 2008) and knowledgebased design (VDI5610-2, 2017), which are possible to handle design changes as long as the design logic is clearly understood prior to the modeling tasks. In this regards, the designer must previously examine the design task to define which parts are to be represented and to determine the sequence of operations needed to create these parts. This modeling logic is sometimes visible in auxiliary geometric elements present in the model or in the layers that organize the model but, in most cases, it is "rarely recoverable and difficult to decipher" (Park and Holt, 2010). That means that even slightly design changes often require inordinate amounts of work, as very little modeling logic can be reused. As a result, the traditional design loop is limited by the few solutions that are generated, among which one solution is selected (Sunnersjo, 2016). In fact, in the traditional use of CAD modeling approaches, just a few solutions are represented and the exploration of different solutions requires manual changes to the model. Hence, it is needed a robust design approach to explore a large design solution space and then generate dynamic design artifacts that can be easily adapted to satisfy changes and increases the number and diversity of design solutions.

Generative Design Approach (GDA) adopted in this research originates from Sauthoff (2017) and later further developed by Li and Lachmayer (2018), aiming to support automatic and iterative configuration to generate creative designs by capturing design knowledge via a set of design elements. As a result, GDA is considered as a more efficient approach in CAD model generation. In fact, since GDA provides flexibilities for configuring a CAD model through variable design elements, design solution space is therefore easily explored via iterative design processes in a timely and cost-effective manner. However, there are surprisingly little researches providing detailed exploration process for design solution space. Hence, the purpose of this study is to provide an exploration process in detail, with which a large design solution space can be explored during design, which is robust against design changes.

\subsection{Paper structure}

The structure of the rest paper is reflected as follow: the current researches and the key issues in design solution space exploration, e.g., CAD modeling strategy and design solution space construction, are reviewed in section 2. In section 3, GDA is illustrated and implemented as an exploration method to construct a large solution space of a crank following certain design procedures. In section 4 , an analysis of the performance in design solution space exploration is employed between traditional modeling strategies and GDA, and finally, the associated conclusions and limitations are given. 


\section{CURRENT STATE OF RESEARCH}

To address the definitions and questions of design solution exploration during product development, a literature review was undertaken, in which viable facets including modeling strategy, design solution space construction and exploration were examined.

\subsection{CAD modeling strategy}

CAD modeling is an essential part of engineering design activity via specialized software. The outputs are normally CAD models of an object, which can be used to modify, analyze or optimize the design of the object. Modeling strategy deals with the way, in which objects can be characterized as a unified methodology for capturing and representing product information via CAD modeling software. Usage of CAD modeling strategies decreases the amount of engineering mistakes and makes visualization easier. A review of general 3D product modeling techniques and methods is given in VDI2209 (2009), by the way of introduction, Figure 1 gives an overview of them.

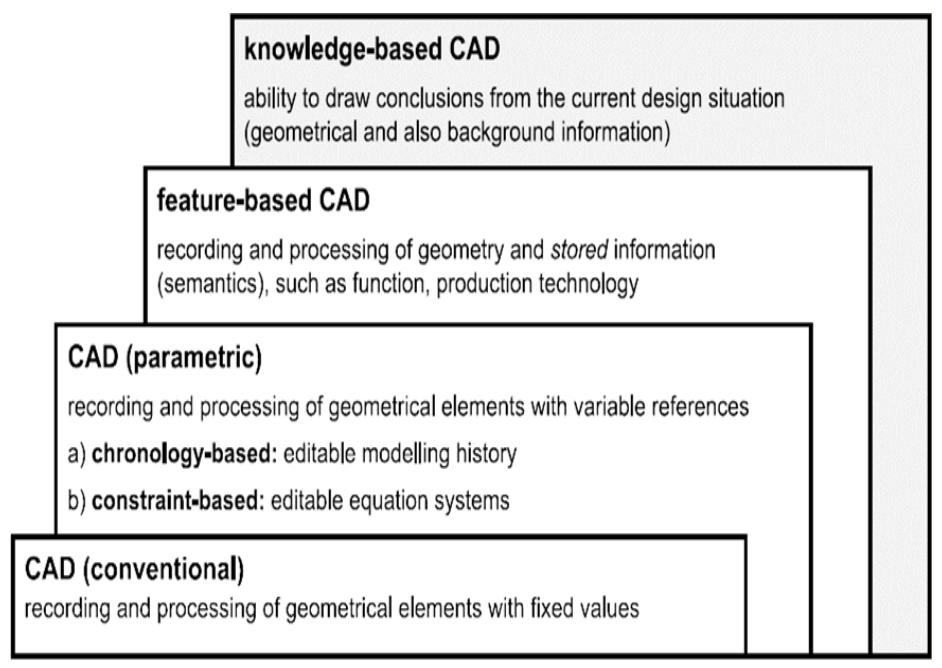

Figure 1. Overview of the principles of 3D modeling (VDI2209 2009)

Conventional modeling (CM), also known as direct modeling, is used to generate models with fixed values during the generation process without the explicit use of parameters or references (Renno and Papa, 2015). However, the resulting models express only a few state points of design solutions that hardly adapt themselves to handle design changes. Consequently, CM is normally out of the designers' consideration in design solution space exploration. Parametric modeling (PM) maintains the design history steps to rebuild the model instead of keeping only the final outcome geometry on one hand. It also defines dependencies of design histories by a set of constraints that allow users to modify a single operation while the system updates the complete geometry in a consistent manner (Jubierre and Borrmann, 2015). Therefore, PM offers potentials in quickly design solution generation to meet exact performance metrics, design aesthetics, and manufacturing criteria (Hoffmann, 2005). However, PM requires engineers to anticipate and define a clear plan of constraints, relations and dependencies in order to ensure that design changes will be reflected in all downstream geometries. Thus, design solution exploration is limited in a small design space as known before, since the small range of parametrics and the relations are also defined in a simple way.

Typically, feature-based modeling (FBM) is developed as a layer on top of an established geometric modeling technique which refers to the construction of geometries as a combination of form features, which preserve design intents of the model as they also carry manufacturing and design creation history information (VDI2218, 2003). Since the final models represent variable requirements in feature levels, describing an aggregation of properties of a specific product, design solution exploration reaches an extended design space out of the known one. Knowledge-based modeling or design (KBD) is a research area for design solution generation that involves complex and iterative processes by capture and reuse of product knowledge (VDI5610-2, 2017). This strategy enables designers to generate design solutions through the flow of knowledge, thus the design requirements are formulated in terms of the latest knowledge and technology. In this regard, the uncertain design requirements are detail synthesized, so as to obtain a greater knowledge of their limits (Verhagen 
et al., 2012). Hence, the design solution space is largely explored by taking the formulated design criteria as a basis. With the advantages and benefits from new techniques or methodologies, desired solutions can be instantiated in a completely new design space (Thompson and Pimentel, 2018).

\subsection{Design solution space}

Design is a problem-solving process and can be described in terms of identification of design constraints or requirements and then exploring the feasible solutions. However, such constraints are of different types, not only modeling restrictions such as size, function, load cases and appearance and specifications but also manufacturing restrictions. During the modeling process, there are two main challenges have to be addressed in generating variable design solutions are that design solution space construction to satisfy multiple design constraints and design solution space exploration to search for the best alternatives in terms of those constraints (Kansakar and Munir, 2018).

\subsubsection{Design solution space construction}

In engineering design, it was generally necessary to identify the design problem, which right now there offers no solution. By analyzing the design problem, the problem space and the corresponding solution space are constructed in terms of identifications of design requirements (Akle et al., 2017). Normally, the problem space can be described as the metaphoric space that contains mental representations of the designer's interpretation of the design requirements and restrictions. Thus, the solution space, on the other hand, can be described as the metaphoric space that contains mental representations of the designers' specific solutions by adopting the suitable modeling approaches, based on which they create models and specifications for building the artifact (Vajna et al., 2010).
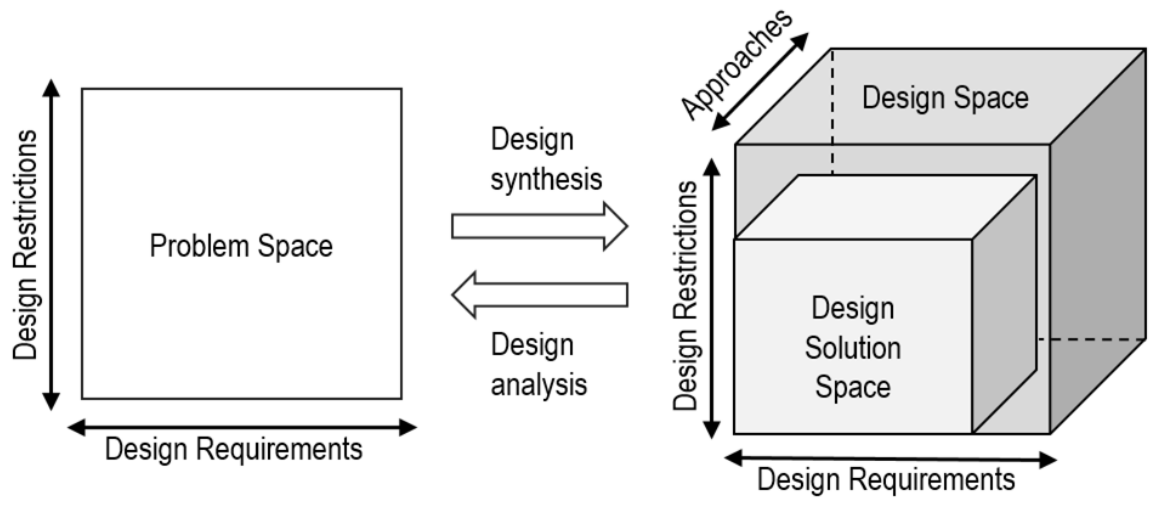

Figure 2. Design solution space construction

As seen in Figure 2, for a design task, design requirements and restrictions are transformed into design constraints that constitute the design problem space where the design objectives are constructed. In terms of such design constraints, designers synthesize those constraints to explore the design conditions and select the suitable modeling approaches that ensure the design space as large as possible. Thus, the set of all solutions that meet those constraints constitute the design space. Design solution space describes the solutions that fit the best, i.e., those with the best performance, from the design space. Later, an analysis process will be carried out so as to prove if the design solution fulfilling all design constraints. As a result, an efficient design solution space is constructed reflecting all design requirements. However, the design solution space is normally smaller than the design space because designers are only able to apply one approach or maximum combine a few of them. For this, a powerful modeling approach should provide stronger supports in design to setup the design solution space as large as possible.

\subsubsection{Design solution space exploration}

Due to the limited information in the early stage of design, the designers have to deal with different types of uncertainty, e.g., uncertainties of design variables and ambiguous design aesthetics (Bodein et al., 2014). Further, with growing interests in the realization of complex design task, there is an increasing need for developing methods to explore the design solution space via CAD models that approximate reality (Gembarski and Lachmayer, 2018). In engineering design practices, a meaningful solution space can be defined by applying variable modeling approaches based on a proper parameterization process of CAD models. Although generating a large set of design alternatives that are meaningful for the design 
constraints to be analyzed is a key step in the design process, it still cannot effectively clarify the suitable design solutions (Li et al., 2018). The identification of suitable design solutions is in fact based on a proper exploration of the solution space of the design task. This includes searching among the design solutions not only for instances that satisfy the traditional design constraints as well as the given engineering specifications but also for principles underlying the design trends, which can be used as feedbacks for the overall design process and for parameterizing and exploring additional models (Turrin et al., 2011). With respect to this situation, Kang et al. (2010) suggest that an effective design solution space exploration framework needs to consist of the following ingredients: (1) a suitable representation of the design space, (2) an effective exploration method, and (3) machine-assisted techniques for analyzing. Related to this framework, the exploration process requires suitable knowledge-integrated designs for representing and visualizing design solutions, which enable the designers to evaluate the suggested solutions.

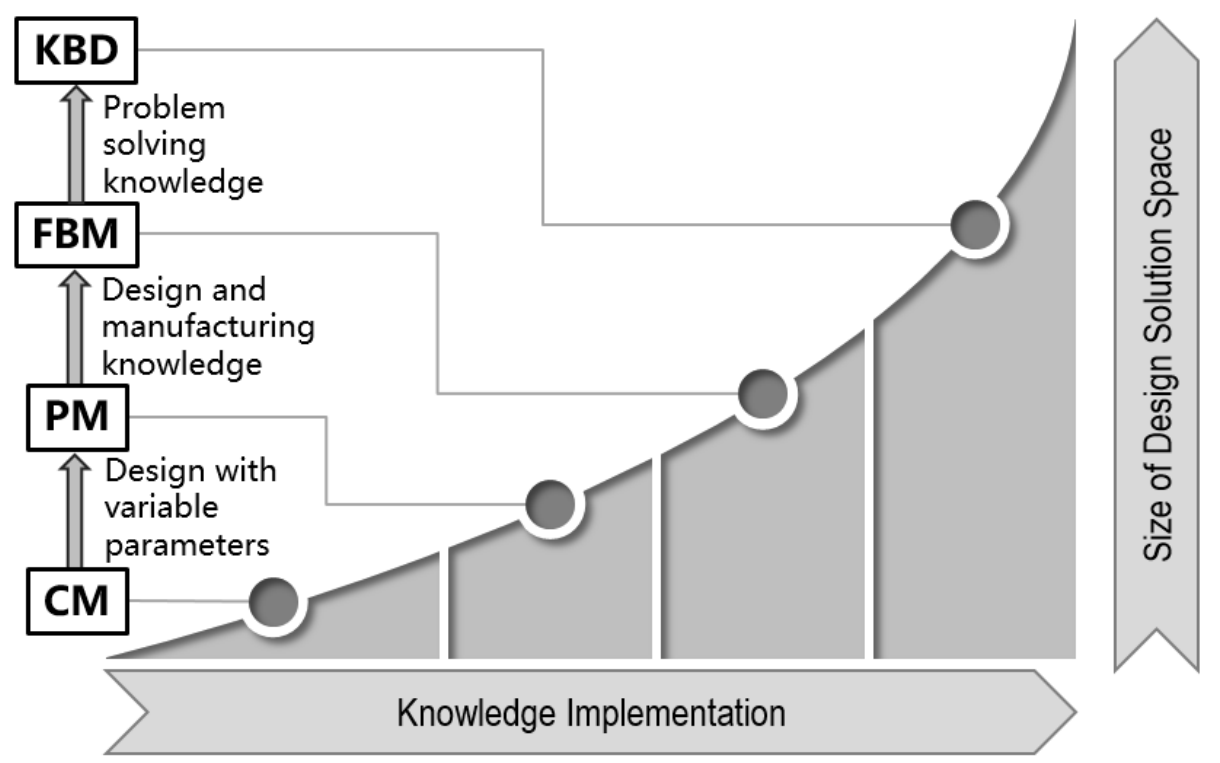

Figure 3. Knowledge-based design solution space exploration

As seen in Figure 3, from CM to KBD, the more design knowledge is implemented, the larger size of the design solution space is explored. Since the design solution space exploration is mainly a knowledgeoriented process, it is possible to represent the inherent knowledge of many design problems through some hierarchical associative constraints, which will provide the guidance of the exploration process for the design solutions (Turrin et al., 2011). Thus, both domain knowledge and control knowledge have to be involved during design, in which the former structures the feasible design solution space, e.g., via a set of constraints or design template while the latter determines the exploration process of design solution space, such as rule-based or case-based exploration (Gembarski and Lachmayer, 2018). Thus, many attempts have been applied in various design problems, such as template-based design (Gembarski et al., 2017) and mechanical systems (Vaillant, 2016). However, there is still a lack of effective means to capture, reuse, and represent variable design knowledge in the exploration process of design solution space. Therefore, an efficient design solution exploration approach is a must because current modeling strategies simulate all the possible configurations in a large design space is not temporally feasible.

\section{GENERATIVE DESIGN APPROACH IMPLEMENTATION}

GDA is a comprehensive CAD based generative design exploration method designed to work at all stages during the product development process. With such an approach, different design applications can be developed by using the same system prototype, but with different initial design representations and selections of transformation methods ( $\mathrm{Li}$ and Lachmayer, 2018). The basic idea behind is to divide a product into several design elements independently after structuring and analyzing its functionalities. These design elements are configured together via a design skeleton and references of connecting interfaces. This configuration of design elements enable designers to generate a large number of design solutions that are robust against design changes. In the end, the exploration of the possible design solutions is automated to find the best solution in terms of evolving design requirements. In summary, 
the main procedure of GDA implementation to explore and generate a large number of design solutions consists of 3 steps: 1) define a design skeleton after separation; 2) create a knowledge-integrated design element database; and 3) select and configure the desired design elements to form a product.

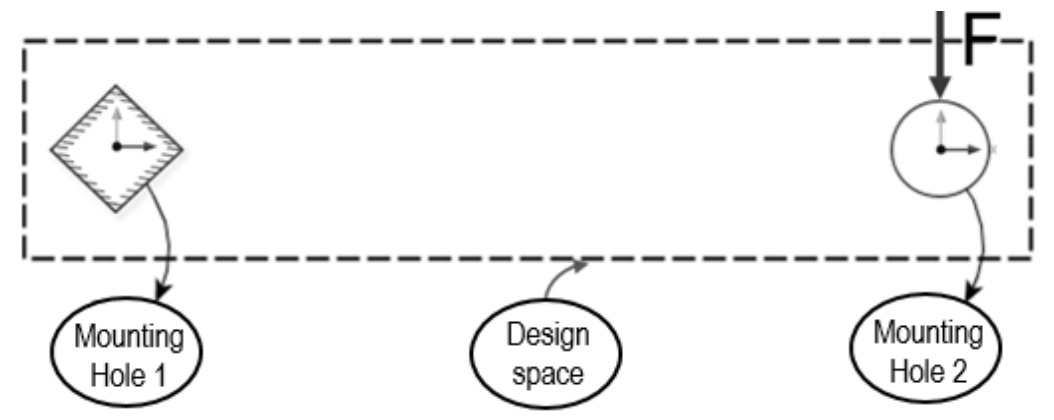

Figure 4. Crank design

For the demonstration of the approach, this study takes a crank as a running example. The basic concept of such crank consists of two mounting holes, in which the mounting hole 1 is fixed and the mounting hole 2 has another design with a load F (Figure 4). For that, the main problem is how to balance the weight and structural stiffness of such crank, and the other requirements, e.g., the cost and aesthetic of the crank, have to be considered as well.

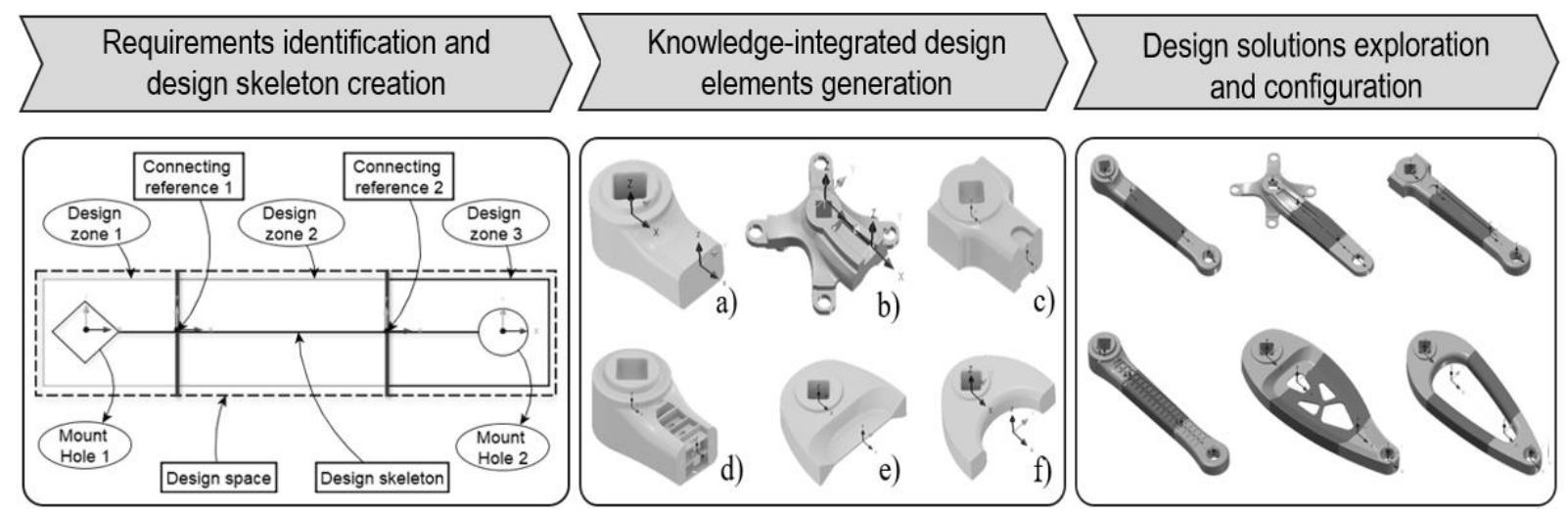

Figure 5. Crank design process by GDA

The process of GDA starts with a designer defining a design skeleton referred to the separation of the crank via a set of parameters, constraints and connecting points (Figure 5). As shown in Figure 4, the mounting holes are the most important criteria that have to be considered. According to its structure and functions, this crank is divided into 3 design zones, so that the resulting skeleton is defined by 4 local coordinate systems that address the assembly positions as well as the orientations of all 3 design zones. Likewise, the connecting points are also defined at every local coordinate systems so as to set a reference for modeling and placing different design elements. Based on this skeleton, each design zone of the crank has a unique modeling structure, which is already linked to neighboring design zones.

With respect to the second step, a creation example of the design elements of design zone 1 is presented (Figure 6). As introduced in section 2.2.2, both domain knowledge and control knowledge of the crank have to be implemented. In this case, different design elements integrated design knowledge are realized through following points: numbers of design zones to setup design elements, the diversity types and arrangements of connecting interfaces, e.g., pure circle or combine circle and rectangle, and the applied design concepts, e.g., with or without screw. In this way, design knowledge is transformed into a series of design rules, constraints or parameters that can be understood by CAD systems. When a design element is established, a check is made by design requirements and design guidelines to verify if the current set of parameters is allowed or conflicting. A design element is finished if all parameters are solved and without conflicts with the requirements on the weight and stiffness of the crank. Thus, the knowledge related to the crank is translated to a set of formulas and rules that describe its behaviors. As a result, the designed elements have the same function, but different topologies are described in detail for the purpose of covering the allowable design space. Finally, design solution space exploration is transformed as a configuration task that can be automated in CAD systems. 


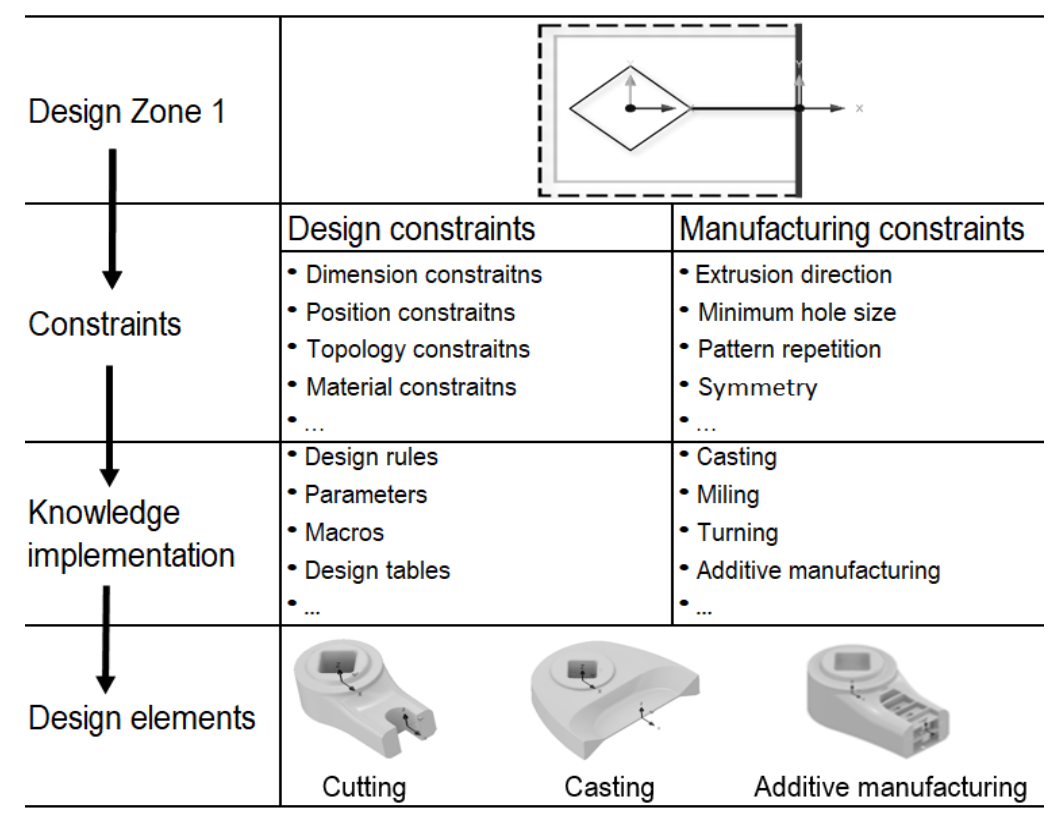

Figure 6. Knowledge-integrated design elements generation process

The last operation of the crank generation is design elements selection and configuration. Figure 7 shows the generation process of the crank. In order to achieve a user-friendly dialog, Autodesk Inventor CAD system is extended with a CAD plug-in application that supports the generation of such a crank. In this application, the design rules behind will retrieve the corresponding design elements and configure those at the right place via the selection of connecting interfaces, which is basically done by inheriting parameters of the design skeleton to the design elements, where the positions and orientations of design elements are. The local coordinate systems in the design skeleton aid for positioning design element one after another and orient each design element in order to generate a required crank. In this application, different models can be generated by changing the connecting interfaces. At last, the most relevant design solution is identified towards to the design requirements or design changes.

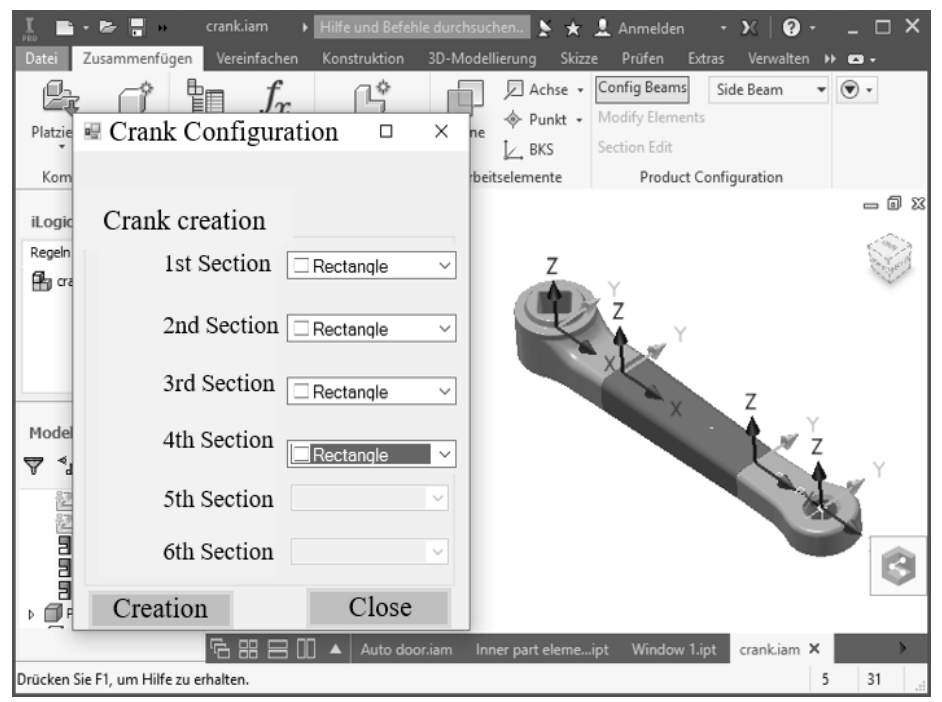

Figure 7. Design elements selection and configuration

In general, GDA allows a designer the flexibility to explore more design solutions than were possible with traditional modeling approaches. By integrating and automating this entire design process, it is possible to create higher quality solutions in much less time. In tandem with this, GDA also makes it simple to explore exactly how design goals are impacted by design changes, find worthwhile, previously unconsidered design configurations, and determine values of design variables required to solve specific problems. Together with FEA and a parameter-based optimization, the exploration of the possible solution space may be widely automated. The result is optimized products that meet all requirements and keep dynamic to handle design changes in the shortest amount of time. 


\section{COMPARISON AND DISCUSSION}

Section 3 highlights the modeling process of GDA in exploring and generating a large number of design solutions automatically. In this section, an analysis of the performance in design solution space exploration is carried out between GDA and the existing modeling strategies as introduced in section 2.1. In general, despite variable design solutions are generated with those modeling strategies regarding various design objectives and constraints, the size of the design solution space differs (Figure 8).

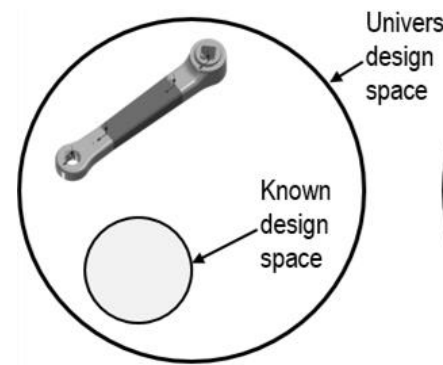

PM

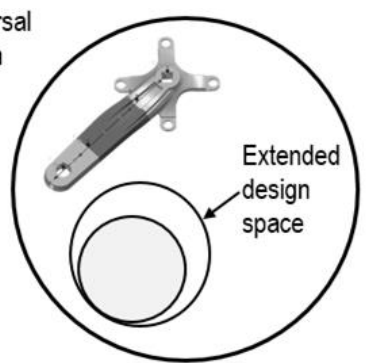

FBM

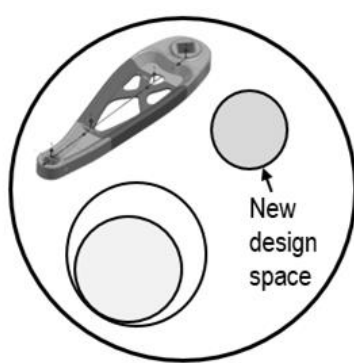

KBD

Figure 8. Crank design solutions by different modeling strategies

With respect to the crank creation, PM normally defines the crank with a set of the design parameters, e.g. length and width, depth and relationships and their applicable ranges, capturing the crank's behaviors and the structures. Therefore, a peddle crank is created. However, due to the limited range of the parameters, the cranks with only a few design variants can only reflect a small design solution space as known before, and the design changes can only happen in modifying the size of the crank and a little bit of the crank's shape. FBM creates a set of design features of the crank that integrate design information and knowledge, e.g., extrusions and holes. In this case, design features with different topologies or shapes are developed and then represent various attributes of the crank, such as the position of the hole. Likewise, the feature order aligns with the steps to create the crank, which also allows modeling the relationships between requirements, functional descriptions and physical solutions of the crank. As a result, an extended design solution space is realized and the design outputs are cranks with variable structures since the values and structures of the defining features are dissimilar. In this context, features are understood as vehicle to bring design knowledge to the downstream design processes for design changes adaptation. However, FBM integrates constraints on features but normally without ontological descriptions of what features are meant to be during design process. As a result, the selection of design features to adjust design changes is still a challenge in design.

KBD incorporates the latest inventions and techniques or ongoing understanding to generate design solution and produce the outputs in a novel manner. Here, a creative crank is realized via the combination of generative design and 3D printing technology. Generative design technology enables designers to implement different innovative inner structures, allowing to simultaneously explore and generate cranks based on design and manufacturing requirements (Figure 9). 3D printing as the manufacturing technology to produce such cranks provides enough stiffness with creative structure. Thus, the design solution space differentiates others by describing a new space out of the known solution space. However, KBD requires each design crank should represent a region of design possibilities, otherwise designers are lost in selecting the appropriate solution regarding design changes.
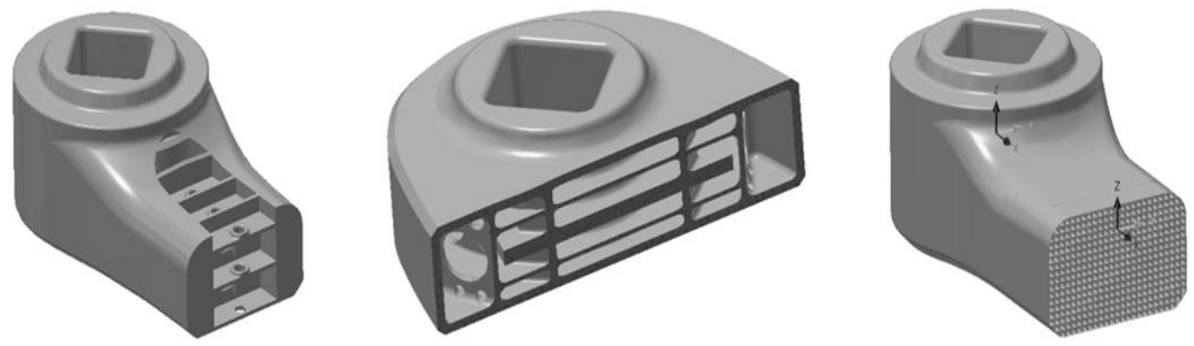

Figure 9. Different inner structures for designing cranks (adapted from Lippert (2018))

Compared to those traditional modeling strategies, GDA can be defined as the creation of design solutions determined by knowledge, focusing on the part level that design problems are transformed 
into a design configuration problem, where design solution space is explored throughout the development process. It does not only save time by exploring and storing all the necessary knowledge of the whole lifecycle through design skeleton and design element database but also integrates CADsystem through friendly user-interfaces that provide reliable technical supports. In addition, the output model is also not only a design solution but also a design concept that the designers could manipulate to facilitate design exploration, because the design elements capture the geometric knowledge and incorporate design restrictions as well. Therefore, the design solution exploration is structured as the search for the variation ranges within the design element database by a set of configuration rules according to the problem space. Especially, designers are challenged in selecting suitable modeling strategies for the accomplishment of a design task in an effective manner, since the size of the solution space in terms of those strategies varies. However, this will never occur by GDA, since this approach already covers design solutions from the known solution space to a completely new one.

Moreover, since GDA provides a set of design elements for every design zone, design changes are therefore not taking place in the whole design model, but on the design elements in the element library, which shortens the adaptation time. During design, the design element library stays dynamic regarding the evolving design requirements or the ongoing understanding or new techniques. That means that the design elements in the element library can be added, modified, replaced or even deleted in order to generate desired design solutions against design changes. In tandem with this, solutions generated via design elements offer interactions among design, industrialization and production, since design elements carried the design knowledge from both design and manufacturing side. This knowledge integration and visibility among design, industrialization and production lead to the reduction of cycle time and improvement of design quality without increasing additional efforts and cost.

A disadvantage, however, is that designers are facing a high demand for knowledge implementation and powerful representation via design elements, since they significantly affect the ability to explore design solutions to some extent. Further, the categorization and organization of the design elements in the database also significantly affect the exploration process, i.e., the effectiveness of design automation.

\section{CONCLUSION}

This research contributed to gain a further understanding of the use of GDA as an efficient approach to design solution space exploration. The explored solution space provides the rationale behind the design of an artifact, explaining not only the design of the artifact but also the reasons behind why it was designed exactly in that manner. Since many types of design solutions can be generated through GDA, design changes will be quickly adapted in a feasible way by either exchanging design elements or modifying design parameters. For assessment, meaningful implementation for a crank creation figured out the role of GDA in task-related design activities by facing evolving design requirements or design changes. Further, during the design process, its effects on the generation of the final design solutions were identified as well. It is confirmed that GDA can be used to complement other approaches such as $\mathrm{PM}, \mathrm{FBM}$ and $\mathrm{KBD}$, providing more concrete aids to overcome the weaknesses observed in design practices at enhancing the diversity and adaptability of the design solutions.

However, limitations of this study should be noted. The implementation of GDA in this research has not considered the uncertainties of design task as well as the complexities of design process. It is possible that the type of design tasks might influence the way that designers dealt with the task, and the limited experience of modeling strategies might also have impacted the design exploration. As a consequence, a comparative study should be carried out firstly, to provide objective and conclusive data that would allow designers to decide which modeling strategy is more efficient in reflecting variable design requirements. Another limitation is the performance of the evaluation method that used to judge the design solutions. Although some check rules have been integrated into design elements that function as judges to evaluate the explored design solutions, there was a high degree of agreement between designers that a powerful evaluation method would enable a better generalization of design solution exploration. Nevertheless, a suitable evaluation method will be developed in the future to suggest which design solution is better than others.

\section{REFERENCES}

Akle, A.A., Yannou, B. and Minel, S. (2017), "Design space visualization for efficiency in knowledge discovery leading to an informed decision”, 21 st International Conference on Engineering Design (ICED17). 
Alfaris, A.A.F., 2009. Emergence through conflict: the Multi-Disciplinary Design System (MDDS), Doctoral dissertation, Massachusetts Institute of Technology.

Bodein, Y., Rose, B. and Caillaud, E. (2014), "Explicit reference modeling methodology in parametric CAD system", Computers in Industry, Vol. 65 No. 1, pp. 136-147. https://doi.org/10.1016/j.compind.2013.08.004.

Gembarski, P., Li, H., Lachmayer, R. (2015), "KBE-Modeling Techniques in Standard CAD-Systems: Case Study-Autodesk Inventor Professional”, In: Bellemare, J., Carrier, S., Nielsen, K., Piller F. (Ed.), Managing Complexity. Springer, Cham, pp. 215-233. https://doi.org/10.1007/978-3-319-29058-4_17

Gembarski, P., Li, H. and Lachmayer, R. (2017), "Template-Based Modelling of Structural Components", International Journal of Mechanical Engineering and Robotics Research, Vol. 6 No. 5, pp. 336-342.

Gembarski, P.C. and Lachmayer, R. (2018), "The Parameter Space Matrix as Planning Tool for Geometry-based Solution Spaces", Proceedings of the 8th International Conference on Mass Customization and Personalization - Community of Europe (MCP - CE 2018), Novi Sad, Serbien, 19.09.-21.09.2018.

Hoffmann, C. (2005), "Constraint-based computer-aided design”, Journal of Computing and Information Science in Engineering, Vol. 5 No. 3, pp. 182-187. https://doi:10.1115/1.1979508.

Jubierre, J. and Borrmann, A. (2015), "Knowledge-based engineering for infrastructure facilities: assisted design of railway tunnels based on logic models and advanced procedural geometry dependencies", Journal of Information Technology in Construction, Vol. 20 No. 26, pp. 421-441.

Kang, E., Jackson, E. and Schulte, W. (2010), “An approach for effective design space exploration”, Monterey Workshop (pp. 33-54). Springer, Berlin, Heidelberg. https://doi.org/10.1007/978-3-642-21292-5_3.

Kansakar, P. and Munir, A. (2018), “A Design Space Exploration Methodology for Parameter Optimization in Multicore Processors", IEEE Transactions on Parallel and Distributed Systems, Vol. 29 No. 1, pp. 2-15. https://doi.org/10.1109/tpds.2017.2745580. .

Li, H. and Lachmayer, R. (2018), "Generative Design Approach for Modeling Creative Designs”, In IOP Conference Series: Materials Science and Engineering, Vol. 408 No. 1, pp. 012035. https://doi.org/10.1088/1757-899X/408/1/012035.

Li, H., Gembarski, P.C. and Lachmayer, R. (2018), “Template-based design for design co-creation”, In DS 89: Proceedings of The Fifth International Conference on Design Creativity (ICDC 2018), University of Bath, Bath, UK (pp. 387-394).

Lippert, B. (2018), Restriktionsgerechtes Gestalten gewichtsoptimierter Strukturbauteile für das Selektive Laserstrahlschmelzen, $\mathrm{PhD}$ thesis, Leibniz Universität Hannover.

Renno, F. and Papa, S. (2015), "Direct Modeling Approach to Improve Virtual Prototyping and FEM Analyses of Bicycle Frames", Engineering Letters, Vol. 23 No. 4.

Sauthoff, B. (2017), Generative Parametrische Modellierung von Strukturkomponenten für die Technische Vererbung, $\mathrm{PhD}$ thesis, Leibniz Universität Hannover.

Shahin, T. (2008), "Feature-based design - an overview", Computer-Aided Design and Applications, Vol. 5 No. 5, pp. 639-653. https://dx.doi.org/10.3722/cadaps.2008.639-653.

Sunnersjo, S. (2016), "Industrial Products and How They Are Developed", In: Intelligent Computer Systems in Engineering Design, Springer, Cham. https://doi.org/10.1007/978-3-319-28125-4.

Thompson, M. and Pimentel, A.D. (2013), "Exploiting domain knowledge in system-level MPSoC design space exploration", Journal of Systems Architecture, Vol. 59 No. 7, pp. 351-360. https://doi.org/10.1016/j.sysarc.2013.05.023.

Turrin, M., Von Buelow, P. and Stouffs, R. (2011), "Design explorations of performance driven geometry in architectural design using parametric modeling and genetic algorithms", Advanced Engineering Informatics, Vol. 25 No. 4, pp. 656-675. https://doi.org/10.1016/j.aei.2011.07.009.

Park, K. and Holt, N. (2010), "Parametric design process of a complex building in practice using programmed code as master model”, International Journal of Architectural Computing, Vol. 8 No. 3, pp. 359-376. https://doi.org/10.1260/1478-0771.8.3.359.

Vaillant, M. (2016), Design Space Exploration zur multikriteriellen Optimierung elektrischer Sportwagenantriebsstränge, $\mathrm{PhD}$ thesis, Karlsruher Institut für Technologie.

Vajna, S., Kittel, K. and Bercsey, T. (2010), "Designing the solution space for the autogenetic design theory (ADT)”. In DS 60: Proceedings of DESIGN 2010, Dubrovnik, Croatia (pp. 1441-1450).

VDI 2209 (2009), 3D product modelling. Technical and organizational requirements, Beuth Verlag, Berlin.

VDI 2218 (2003), Information technology in product development: feature technology, Beuth Verlag, Berlin.

VDI 5610-2 (2017), Knowledge management for engineering: knowledge-based engineering, Beuth Verlag, Berlin.

Verhagen, W., Bermell-Garcia, P., van Dijk, R. and Curran, R. (2012), “A critical review of Knowledge-Based Engineering: An identification of research challenges", Advanced Engineering Informatics, Vol. 26 No. 1 , pp. 5-15. https://doi.org/10.1016/j.aei.2011.06.004.

Weber, C. (2011), "Design Theory and Methodology-Contributions to the Computer Support of Product Development / Design Processes”, In: The Future of Design Methodology (pp. 91-104), Springer, London. 